\title{
Pulmonary embolism diagnosed immediately following blunt trauma
}

\author{
Catherine R Lewis, Mustafa K Davis, Omar K Danner, \\ Kenneth L Wilson, L Ray Matthews
}

\begin{abstract}
Introduction: Physicians who care for trauma patients should be aware of the possibility of a pulmonary embolism presenting immediately after traumatic injury. Case Report: We report a case of a 30-year-old female restrained driver who developed a pulmonary embolism within two hours of a motor vehicle accident. Conclusion: We conclude that trauma can produce a hypercoagulable state that can occur immediately after a traumatic event and result in a pulmonary embolism.
\end{abstract}

Keywords: Pulmonary embolus, Trauma, Hypercoagulable state, Embolism

$* * * * * * * * *$

Lewis CR, Davis MK, Danner OK, Wilson KL, Mattews LR. Pulmonary embolism diagnosed immediately following blunt trauma. International Journal of Case Reports and Images 2013;4(5):248-251.

$* * * * * * * * *$

Catherine R Lewis ${ }^{1}$, Mustafa K Davis², Omar K Danner³, Kenneth L Wilson ${ }^{3}$, L Ray Matthews ${ }^{3}$

Affiliations: ${ }^{1}$ Morehouse School of Medicine, PGY-1, Department of Surgery, Atlanta, GA, USA; ${ }^{2}$ Morehouse School of Medicine, PGY-IV, Department of Surgery, Atlanta, GA, USA; ${ }^{3}$ Morehouse School of Medicine, Assistant Professor, Department of Surgery, Atlanta, GA USA.

Corresponding Author: L Ray Matthews, Morehouse School of Medicine, 720 Westview Drive SW, Atlanta, GA, USA. 30310; Ph: 404-616-2391, 404-616-1417; Email: lematthews@msm.edu

Received: 21 January 2012

Accepted: 22 March 2012

Published: 01 May 2013
doi:10.5348/ijcri-2013-05-305-CR-2

\section{INTRODUCTION}

Pulmonary embolism (PE) is very rare immediately after a traumatic event and has been reported only once in the literature. Pulmonary embolism is usually a complication that on average, occurs 4-7 days after serious injury [1]. However, there is still no definitive explanation for the cause and timing of PE in trauma patients. Several mechanisms for pulmonary embolism have been proposed, although it is recognized that coagulation and fibrinolytic systems are triggered immediately after trauma due to direct tissue injury [2]. Several risk factors have been identified for the development of pulmonary embolism. Physicians who care for trauma patients should be aware of the possibility of a pulmonary embolism presenting immediately after traumatic injury. We report a case of a PE that developed within two hours of a motor vehicle accident. As hypercoagulability is present acutely posttrauma, this may explain the almost immediate development of a pulmonary embolism in our patient who presented with severe head injury. We conclude that trauma produces a hypercoagulable state that can occur immediately after a traumatic event and result in a pulmonary embolism .

\section{CASE REPORT}

A 30-year-old female restrained driver presented to the emergency department after a head-on, rollover motor vehicle collision. The patient was unconscious and found upside down after a prolonged extrication. Primary survey was intact and glascow coma score was 9 (E2, V2, M5). Vital signs on admission were BP, 94/50 mmHg; pulse, 63 beats/min, respirations 13/minute and oxygen saturation $100 \%$ on supplemental oxygen via facemask. Secondary survey revealed the following injuries: a four cm laceration to the posterior, left ear 

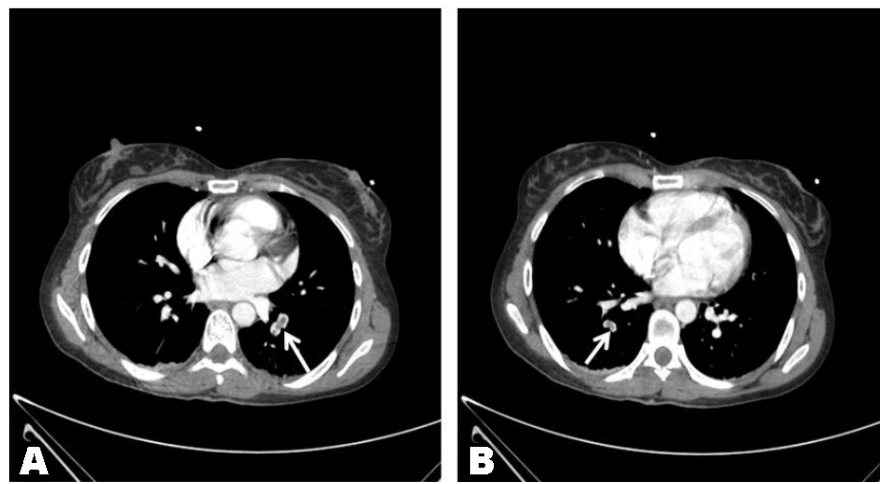

Figure 1: Chest computed tomography scan performed one hr and 33 min after injury. Arrows demonstrate, filling defects in the (A) left main pulmonary artery, and (B) segmental branches of the right, lower lobe.

and a $10 \mathrm{~cm}$ deep laceration to the left, posterior thigh that tracked superiorly. Cardiac and respiratory examinations were within normal limits. The patient's past medical history was unremarkable as it related to bleeding disorders and there was no previous history of smoking. There was a reported use of oral contraceptives for one month prior to presentation.

Chest and pelvic radiographs were negative for acute injuries. Computed tomography (CT) of the head, cervical spine, chest, abdomen and pelvis were performed within two hours of initial injury. Head CT scan demonstrated multiple, small, acute hemorrhagic contusions in the frontal and temporal lobes. Chest CT was significant for bilateral pulmonary emboli in the left and right lower lobes (Figure 1).

Initial laboratory values were all within normal limits. Arterial blood gas on admission, and on supplemental oxygen, was pH 7.36; $\mathrm{PaO} 2424 \mathrm{mmHg}$, $\mathrm{PaCO} 238 \mathrm{mmHg}$, and $\mathrm{SaO}_{2}$ 98\%. Transthoracic echocardiogram showed mild septal wall hypokinesis and abnormal motion in the distal anterolateral segment consistent with myocardial contusion. There was no evidence of right heart strain. Doppler ultrasound of the lower extremities was negative for deep vein thrombosis.

Systemic anticoagulation and/or thrombolysis were contraindicated because of the risk of blossoming of the multiple cerebral contusions. Cardiothoracic surgery consultation deemed that the thrombus was situated too distally in the left pulmonary artery and was not easily accessible for possible embolectomy. The patient was therefore treated on hospital day-2 with placement of a Cook Celect ${ }^{\mathrm{TM}}$ Vena Cava Filter. The patient was initially admitted to the surgical intensive care unit. After stabilization of her head injury, the patient was transferred to the surgical floor on hospital day-4. On hospital day-6, the patient was transferred to an acute traumatic brain injury rehabilitation center without complications.

\section{DISCUSSION}

Virchow's triad of stasis, vascular damage and hypercoagulability causing thromboembolism was described over a century ago [3]. However, there is still no definitive explanation for the cause and timing of $\mathrm{PE}$ in trauma patients. Several mechanisms have been described, which guide prophylaxis and treatment of PE in trauma patients. Coagulation and fibrinolysis are triggered immediately after trauma due to direct tissue injury $[4,5]$. Hemorrhage, shock, hypoxia, fat embolism and infection are examples of conditions which in almost all trauma patients predispose to the development of thromboembolism [4]. It has also been proposed that fibrin degradation products are elevated immediately after trauma and gradually return to normal over several days [5]. However, many laboratory values that are measured to determine a hypercoagulable state are inaccurate in the setting of trauma as a result of active resuscitation and ongoing blood loss which may distort the results.

What is known about the process of coagulation and fibrinolysis has led to the identification of several risk factors for the development of pulmonary embolism. Spinal cord injuries and spinal fractures place the patients at high risk for the development of PE [6]. Age, pelvic fractures, multiple long bone fractures and central vein cannulation are also known risk factors [6]. The incidence of $\mathrm{PE}$ was shown to be $0.5 \%$ in high risk patients (multiple fractures or spinal cord injuries) and $0.2 \%$ in patients not at an increased risk [6]. Attar et al., analyzed four groups of acute trauma patients and determined that those with craniocerebral trauma had a more persistent state of hypercoagulability as compared to the other groups, which was thought to be due to hypothalamic stimulation [4]. As hypercoagulability is present immediately post-trauma, this may explain the almost immediate development of a PE in our patient who presented with traumatic brain injury.

The most common signs and symptoms of $\mathrm{PE}$ include dyspnea and tachypnea. However, the symptoms are nonspecific and may range from an asymptomatic presentation to sudden death. As in our patient and most trauma patients, it is often difficult to diagnose a PE because of altered mental status, intubation or other injuries that may explain the symptoms of PE [5-7]. Traditionally, it has been taught that a PE is most likely to occur 5-7 days after injury [7]. This makes it likely for many trauma physicians to overlook PE presenting along with the injury. However, studies have shown that up to $25 \%$ of PE's may occur within the first four days after injury [7], with one author reporting diagnosis of PE within several hours after injury [7]. Thrombosis from a direct vessel injury could not be excluded in that particular case as their presentation involved a patient with an associated aortic injury $[6,7]$. We report an even earlier diagnosis of a PE, one hour and 33 minutes after initial injury. Therefore, it is important to recognize that $\mathrm{PE}$ should not be excluded as an early diagnosis in trauma patients. 
Thrombi of the deep venous system may form during the course of an elective surgical procedure, warranting the use of prophylactic measures prior to anesthesia induction [7]. However, prophylactic measures are not always possible for trauma patients and/or may be contraindicated based on their injuries. Thromboembolism prophylaxis is imperative for all trauma patients as soon as possible after injury. Owings et al. demonstrated that delaying or withholding prophylaxis resulted in an earlier diagnosis of PE [2]. At our institution, we routinely use sequential compression devices, in addition to chemical prophylaxis upon admission. In patients with absolute contraindications to anticoagulation or in those with prolonged immobilization, we may also elect to place a vena cava filter. Embolectomy was not a surgical option in our patient due to the location of the thrombus and thrombolysis would have been risky given her cerebral hemorrhage. Embolectomy, thrombolysis and placement of a vena cava filter are all additional options available for management of a PE, and their use must be individually directed on a case-by-case basis. We chose to place a vena cava filter to prevent the development of further PE's and to do the safest procedure for the patient based on the given situation.

Trauma physicians should be aware of the possibility of a PE presenting immediately after traumatic injury. Although the typical signs and symptoms of a PE may be explained by additional traumatic injuries or may even be absent, PE cannot be excluded. As a result, it is important to begin prophylaxis as soon as possible to avoid the development of further PE's and additional complications, particularly when findings consistent with PE are identified on initial chest computed tomography scan.

\section{CONCLUSION}

We conclude that trauma produces a hypercoagulable state that can occur immediately after a traumatic event and result in a pulmonary embolism.

$* * * * * * * *$

\section{Author Contributions}

Catherine R Lewis - Substantial contributions to conception and design, Acquisition of data, Analysis and interpretation of data, Drafting the article, Revising it critically for important intellectual content, Final approval of the version to be published

Mustafa K Davis - Acquisition of data, Drafting the article, Revising it critically for important intellectual content, Final approval of the version to be published Omar K Danner - Acquisition of data, Drafting the article, Revising it critically for important intellectual content, Final approval of the version to be published Kenneth L Wilson - Acquisition of data, Drafting the article, Revising it critically for important intellectual content, Final approval of the version to be published
L Ray Matthews - Acquisition of data, Drafting the article, Revising it critically for important intellectual content, Final approval of the version to be published

\section{Guarantor}

The corresponding author is the guarantor of submission.

\section{Conflict of Interest}

Authors declare no conflict of interest.

\section{Copyright}

(C) Catherine $\mathrm{R}$ Lewis et al. 2013; This article is distributed under the terms of Creative Commons attribution 3.0 License which permits unrestricted use, distribution and reproduction in any means provided the original authors and original publisher are properly credited. (Please see www.ijcasereportsandimages.com /copyright-policy.php for more information.)

\section{REFERENCES}

1. Menaker J, Stein DM, Scalea TM. Incidence of early pulmonary embolism after injury. J Trauma 2007;63(3):620-4.

2. Owings JT, Kraut E, Battistella F, Cornelius JT, O'Malley R. Timing of the occurrence of pulmonary embolism in trauma patients. Arch Surg 1997;132(8):862-6.

3. Rogers FB, Osler TM, Shackford SR. Immediate pulmonary embolism after trauma: case report. J Trauma 2000 Jan;48(1):146-8.

4. Attar S, Boyd D, Layne E, McLaughlin J, Mansberger $\mathrm{AR}$, Cowley RA. Alterations in coagulation and fibrinolytic mechanisms in acute trauma. J Trauma 1969;9(11):939-65.

5. Schmidt U, Enderson BL, Chen JP, Maull KI. Ddimer levels correlate with pathologic thrombosis in trauma patients. J Trauma 1992;33(2):312-9; discussion 319-20.

6. Flute PT. Coagulation and fibrinolysis after injury. J Clin Path. J Clin Pathol Suppl (R Coll Pathol) 1970;4:102-9.

7. O'Malley KF, Ross SE. Pulmonary embolism in major trauma patients. J Trauma 1990;30(6):748-50. 
Access full text article on

other devices

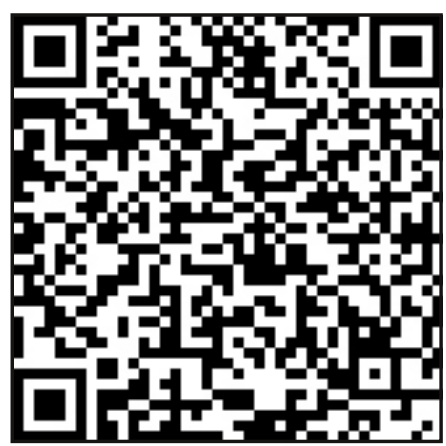

Access PDF of article on other devices

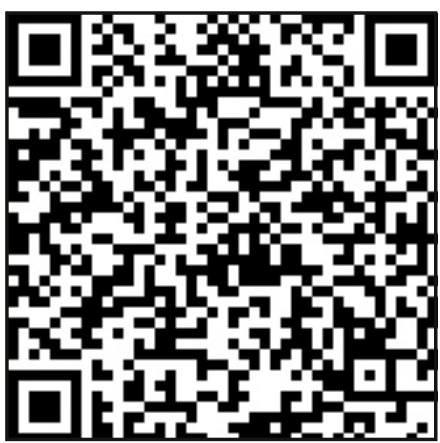

